\title{
The Study of Dynamic Characteristic of Acupoints Based on the Primary Dysmenorrhea Patients with the Tenderness Reflection on Diji (SP 8)
}

\author{
Sheng Chen, ${ }^{1}$ Yanhuan Miao,, Yinan Nan, ${ }^{3}$ Yanping Wang, ${ }^{2}$ Qi Zhao,, \\ Enhui He, ${ }^{1}$ Yini Sun, ${ }^{1}$ and Jiping Zhao ${ }^{1}$ \\ ${ }^{1}$ Acupuncture and Moxibustion Department, Dongzhimen Hospital, Beijing University of Chinese Medicine, \\ No. 5 Haiyuncang, Dongcheng District, Beijing 100010, China \\ ${ }^{2}$ College of Acupuncture-Moxibustion and Tuina, Beijing University of Chinese Medicine, No. 13 of the North 3rd East Road, \\ Chaoyang District, Beijing 100029, China \\ ${ }^{3}$ International Medical Center, China-Japan Friendship Hospital, No. 2 Yinghua East Street, Chaoyang District, Beijing 100029, China
}

Correspondence should be addressed to Jiping Zhao; zjp7883@sina.com

Received 19 December 2014; Revised 3 March 2015; Accepted 3 March 2015

Academic Editor: Haifa Qiao

Copyright (C) 2015 Sheng Chen et al. This is an open access article distributed under the Creative Commons Attribution License, which permits unrestricted use, distribution, and reproduction in any medium, provided the original work is properly cited.

In TCM theory, acupoint is not a fixed point but a portal with dynamic characteristics where the channel qi enters and flows out. The dynamic characteristics have been verified primarily by detecting the tenderness reaction on Diji (SP 8) in primary dysmenorrhea patients. In this study, finger pressing and algometer were applied in Diji (SP 8) area of participants in menstrual period and nonmenstrual period, respectively, to detect the tenderness occurrence rate, the VAS score of the tenderest point, the tenderness threshold of the tenderest point, and the location of the tenderest point. The result suggests that the acupoint may not be a fixed location but a point in a dynamic state within a certain range in time and space varying with different physiological and pathological status.

\section{Background}

Acupoints are specific locations where $q i$ and blood of meridians and Zang-fu organs infuse and also where diseases are reflected and the acupuncture needles are applied [1]. Therefore, acupoint is the essential factor in acupuncture diagnosis and treatment. Each acupoint has its own name, location, specificity, function, and so forth, while location is considered the most essential and fundamental. Without accurate and precise location, it is likely neither to examine the reaction of the acupoint in pathological condition, nor to find the point with therapeutic effects. Because of this, the standardization of the acupoint location is considered as the key in the history of Chinese acupuncture and moxibustion standardization. Since the official promulgation of the location of acupoints (national standard) in 1990, updated standards of the acupoint locations continued to be introduced one after the other in the years 2006 and
2010. The standardization of the acupoint location has to some extent normalized clinical operation and promoted the dissemination and development of the acupuncture and moxibustion worldwide. However what catches our attention is that while promoting the standard of the acupoint location, parts of the clinical practitioners stick too much to the fixed location of the acupoint and ignore the importance and necessity of searching and seeking acupoint along the meridian, leading to dissatisfactory clinical effect.

According to the description in the Yellow Emperor's Inner Canon, the classic of Traditional Chinese Medicine, acupoint is a portal where the channel $q i$ enters and flows out and not a fixed point attaching to the skin, vessel, muscle, tendon, and bone. Therefore, acupoint is not isolated or static structure in the body. It is related to the movement of channel $q i$. It is not only able to reflect the changes of $q i$ in channel, but also able to be used for adjusting the channel qi, showing dynamic characteristics. Whereas most of the researches on 
TABLE 1: Comparison ages between observation group and control group (unit: age).

\begin{tabular}{|c|c|c|c|c|}
\hline \multirow{2}{*}{ Group } & \multirow{2}{*}{ Number of cases } & \multicolumn{3}{|c|}{ Age } \\
\hline & & Smallest value & Largest value & $\mathrm{M}(\mathrm{QR})$ \\
\hline Observation group & 30 & 21 & 34 & $26(5)$ \\
\hline Control group & 30 & 23 & 32 & $25(2)$ \\
\hline$Z$ & & & & -0.88 \\
\hline$P$ & & & & 0.378 \\
\hline
\end{tabular}

dynamic performance of acupoints have been theoretical, clinical research is lacking.

Diji (SP 8) is the most important and commonly used point for the treatment of dysmenorrhea. According to the theory of traditional Chinese medicine (TCM), Diji (SP 8) is the $X i$-cleft point of the Spleen meridian of Foot Taiyin, where the meridian $q i$ accumulates deeply and is suitable for treating acute pain and blood disease. Primary dysmenorrhea, a medical condition of cramping pain in the lower abdomen occurring before and during menstruation, is just ascribed to acute pain and blood disease in TCM. Therefore, this research focuses on patients with primary dysmenorrhea to observe the changes in tenderness in Diji (SP 8) in different physiological and pathological states, so as to explore the dynamic characteristic of acupoint and to provide clinical data for the study of acupoint dynamism from the clinical perspective.

\section{Materials and Methods}

2.1. Setting and Participants. 30 patients with primary dysmenorrhea were recruited as the observation group between April and December of 2013 in Dongzhimen Hospital affiliated to Beijing University of Chinese Medicine. 30 healthy female volunteers from the Beijing University of Chinese Medicine were recruited as the control group during the same period of time.

As some patients with primary dysmenorrhea may resolve or be relieved spontaneously after giving birth, all the participants included were nulliparous, so as to reduce selection bias. In the observation group, the oldest participant was 34 years old, the youngest was 21 years old, and the mean of their age was $26 \pm 5$ years. In the control group, the oldest was 32 years old, the youngest was 32 years old, the smallest age was 23 years, and the mean of their age was $25 \pm 2$ years. After the statistical analysis of the distribution of age and disease duration between the control group and the observation group, the differences were not statistically significant. See details in Table 1.

\subsection{Diagnostic Criteria, Inclusion Criteria, and Exclusion Criteria}

\subsubsection{Observation Group}

(1) Diagnosis Criteria. Referring to the Canadian Department of Gynecology and Obstetrics Association in 2005 primary dysmenorrhea clinical guideline [2], standards are as follows.
(1) The first one is women with lower abdominal pain that begins somewhere between several hours before and a few hours after the onset of the menstrual bleeding, usually persisting up to 2-3 days; (2) the pain is characteristically colicky or dull and located in the midline of the lower abdomen but may extend to both lower quadrants, the lumbar area, and the thighs; (3) the pain is frequently associated with symptoms including diarrhea, nausea, vomiting, fatigue, light-headedness, headache, dizziness, and, rarely, syncope and fever; (4) the symptoms are more or less reproducible from one menstrual period to the other; (5) type B ultrasonic examination and gynecological examination exclude the organic pathological changes in the reproductive organs.

(2) Inclusion Criteria. These criteria include the following: (1) patients who fulfill the diagnostic standard of the primary dysmenorrhea; (2) patients of ages between 18 and 35 years; (3) patients who have never given birth; (4) patients who have disease duration $\geq 6$ months; (5) patients who have regular menstrual cycle $(28 \pm 7) \mathrm{d}$; (6) patients who have abdominal pain which occurs 48 hrs within the onset of menstruation; (7) patients with COX Dysmenorrhea Symptom Scale (CMSS) [3] total score $\geq 8$; (8) patients with VAS score of the abdomen pain $\geq 40$ during the attack of dysmenorrhea; (9) patients who have no participation in any other medication or modality clinical trials; (10) patients who signed informed consent.

(3) Exclusion Criteria. These criteria include the following: (1) patients with life threatening disorders, such as cardiovascular, liver, kidney, hematopoietic system disorders, and mental diseases; (2) patients who have skin problem on and near Diji (SP 8), such as soft tissue damage, ulceration, scar, and skin calluses; (3) patients who received other related treatments within a month or intake of pain killers, sedatives, and hormone drugs within 2 weeks; (4) patients who are physically weak or judged not suitable to participate in this research by researchers.

\subsubsection{Control Group}

(1) Inclusion Criteria. These criteria include the following: (1) healthy women with no abnormalities in the physical examination within the recent semester; (2) patients of ages between 18 and 35 years; (3) patients who have never given birth; (4) patients who have no history of dysmenorrhea in the past; (5) patients who have mostly regular menstrual cycle $(28 \pm 7) \mathrm{d}$; (6) patients who signed informed consent. 


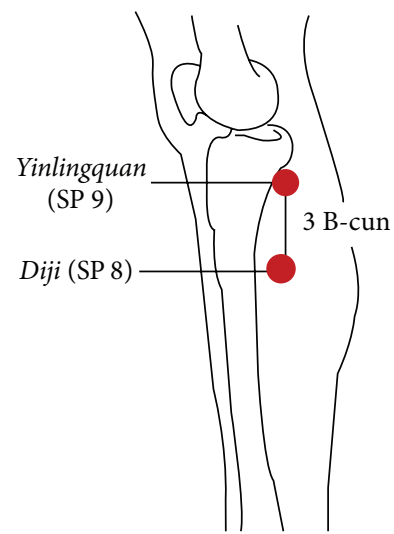

FIGURE 1: Standard position of Diji (SP 8).

(2) Exclusion Criteria. These criteria include the following: (1) patients suffering from frequent lower abdominal pain of unknown reason; (2) patients with skin problems on and near Diji (SP 8), such as soft tissue damage, ulceration, scar, and skin calluses; (3) patients who received other related treatments within a month or intake of pain killers, sedatives, and hormone drugs within 2 weeks; (4) patients with mental disorders or judged not suitable to participate in this research by researchers.

2.3. Tenderness Detection on Diji (SP 8). In order to guarantee the quality of the study, every segment of the operation was performed by the same researcher, who had received training over six months, to ensure the standardization and unity of the operation.

2.3.1. Detecting Point. Diji (SP 8) on both sides of the legs and their surrounding areas of a total of 60 participants in both the observation group and control group was detected.

The participants were instructed to lie supine with legs straightened in a relaxed manner while fully exposing the parts below the knees.

Diji (SP 8) was located referring to WHO standard acupuncture point locations in the Western Pacific Region [4], which is "on the tibial aspect of the leg, posterior to the medial border of the tibia, 3 B-cun inferior to SP9" (Figure 1). Mark this point as the standard position of Diji (SP 8).

2.3.2. Detecting Tenderness with VAS [5]. Starting from the standard position of Diji (SP 8), the researcher pressed spirally with the tip of the thumb pulp in a circular area $2 \mathrm{~cm}$ long in radius. The intensity of the pressing force was consistent and even to the level of muscle. The tenderness was recognized when pain, soreness, or distension sensation was expressed through the immediate and fleeting reactions of participants' eyes or words.

When tenderness reaction appeared, the participants were instructed to face the reverse side of the VAS card without graduation and then move the cursor to the position that best represented the pain intensity. The researcher facing the side with calibration recorded VAS scores and marked

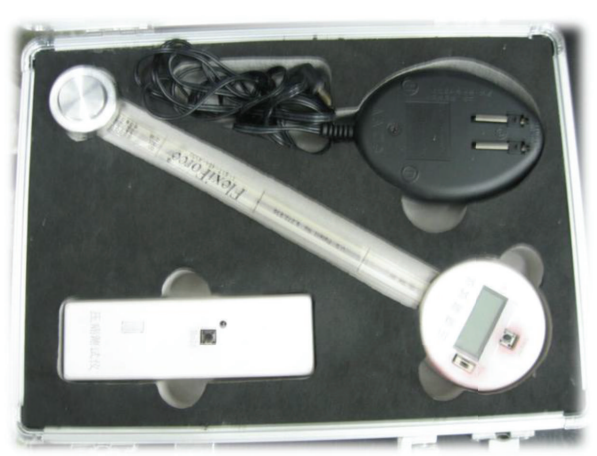

Figure 2: Algometer.

the position. The point with highest VAS scores was the tenderest point; if there is no tenderness upon pressure, the result was just recorded with no VAS detection.

2.3.3. Detecting Pain Threshold of the Tenderest Point. After the VAS assessment, the participants were instructed to rest for 10 minutes. Then, the pain threshold of the tenderest point was detected using an algometer (National Patent number: ZL200520142236.5; Product Publication number: CN2862954; Manufacturer: Institute of Orthopedics and Traumatology Affiliated to Chinese Academy of Traditional Chinese Medicine Science; Place of Production: Beijing) (Figure 2).

Firstly set the tester to zero. Then put the probe tip $(0.5 \mathrm{~cm}$ in diameter) of the tester vertically onto the mark point. Apply pressure gradually and evenly downward (the maximum pressure should not exceed $600 \mathrm{kpa}$ for avoiding tissue damage caused by excessive force). Once the participant reports pain or a radiating pain was elicited, then the algometer was removed and the data on the tester screen was recorded as the pain threshold value. Such a procedure was conducted on both sides of the Diji (SP 8) area, with the left one coming first.

2.3.4. Measure the Location of the Tenderest Point. The distance between the center of the tenderest point and the standard position of Diji (SP 8) was measured with a soft tape measure and then recorded.

\subsubsection{Detecting Time Point}

(1) For the observation group, detection of time point occurred during the first day or second day following the onset of dysmenorrhea and the seventh day after menstruation (nonmenstrual period).

(2) For the control group, detection of time point took place the first or second day following menstrual onset and the seventh day after menstruation (nonmenstrual period).

2.4. Statistical Analysis. SPSS17.0 statistical software was used for analysis. Count data were tested using $x^{2}$ test. Oneway ANOVA was adopted for sets of normally distributed 


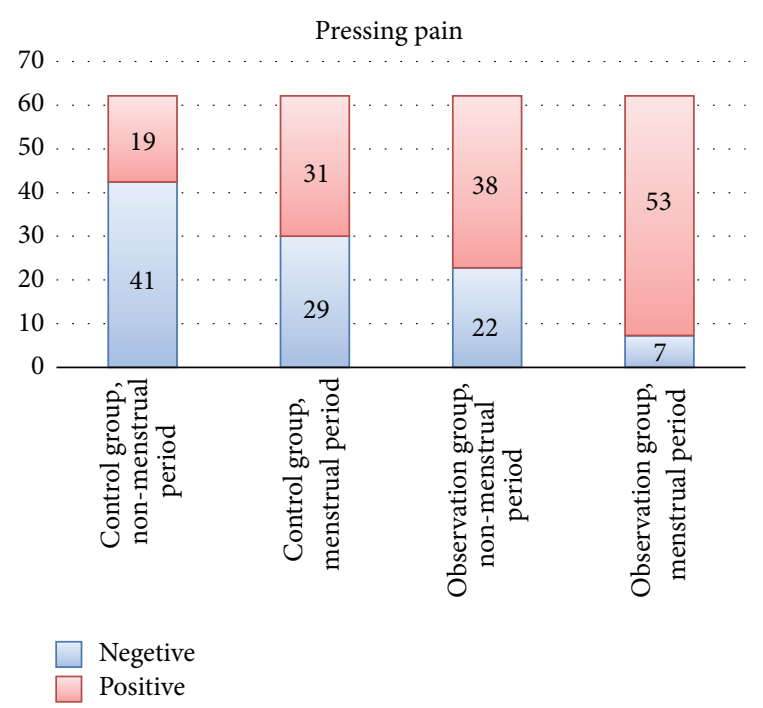

FIGURE 3: Comparison of the TOR of each group during menstrual and nonmenstrual period.

data which went through paired comparison using S-L-D method. $t$-test was used for the data from two groups and the data were expressed by the mean plus or minus standard deviation. Skewed data were tested using the nonparametric Wilcoxon test and expressed in $\mathrm{M}(\mathrm{QR})$, that is, the median (interquartile range). All statistical tests were tested and verified using the two-sided test. $P \leq 0.05$ was considered statistically significant.

\section{Results}

3.1. Comparisons of Tenderness Occurrence Rate in Diji (SP 8) Area. In observation group, there were 5 one-side tenderness cases and 1 pain-free case; the rest of the cases presented with tenderness on both sides in Diji (SP 8) area during acute onset period. Total TOR was $88.3 \%$. During nonmenstrual period, there were 8 one-side tenderness cases and 7 pain-free cases, and the rest presented with tenderness on both sides. Total TOR was $63.3 \%$. In control group, there were 11 oneside tenderness cases and 9 pain-free cases, and the rest of the cases presented with tenderness on both sides in Diji (SP 8) area during menstrual period. Total TOR was 51.7\%. During nonmenstrual period, there were 5 one-side tenderness cases and 18 pain-free cases, and the rest presented with tenderness on both sides. Total TOR was $31.7 \%$.

We used Chi-squared test to compare the TOR between two groups and two menstrual periods (corrected value $P^{\prime}=0.00833$ ). In the observation group, the TOR during menstrual period was significantly higher than that in the nonmenstrual period, $P<P^{\prime}$. In the control group, the TOR during menstrual period was also significantly higher than that in the nonmenstrual period, $P<P^{\prime}$. During nonmenstrual period, the TOR of the observation group was also significantly higher than that of the control group, $P<P^{\prime}$ (Figure 3, Table 2).

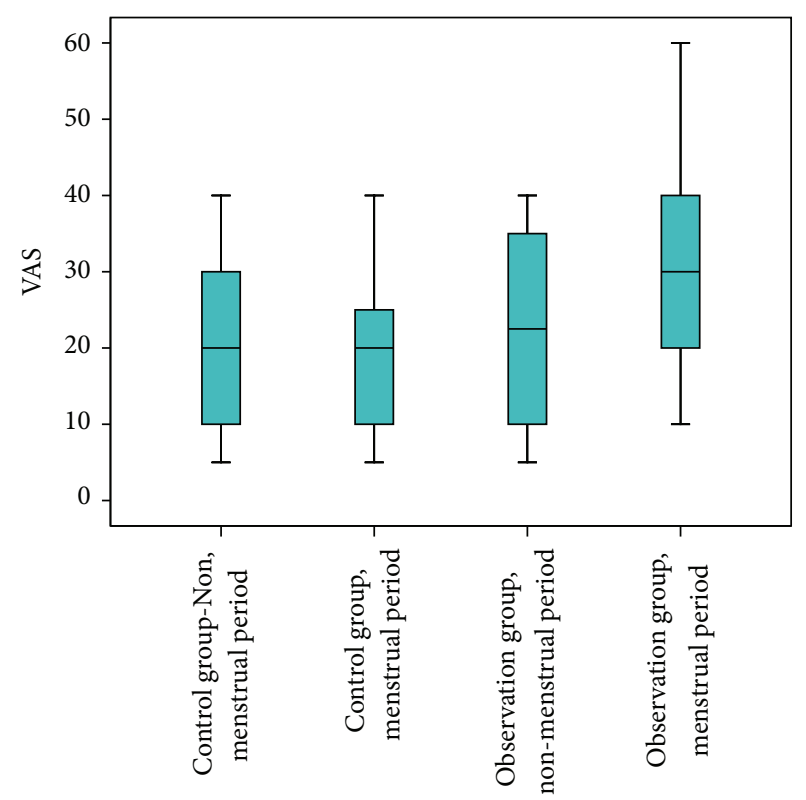

FIGURE 4: Comparison of VAS score between menstrual period and nonmenstrual period in two groups $(\mathrm{mm})$.

3.2. Comparison of VAS Score of the Tenderest Point in Diji (SP 8) Area. Using nonparametric test, we compared VAS score between groups in menstrual period and nonmenstrual period (corrected value $P^{\prime}=0.00833$ ). VAS score of the observation group in the menstrual period was significantly higher than that in the nonmenstrual period, while there was no significant difference between two periods in the control group. In menstrual period, VAS score of the observation group was significantly higher than that of the control group, $P<P^{\prime}$. In nonmenstrual period, VAS score of the observation group was higher than that of the control group, while there was no significant difference, $P>P^{\prime}$ (Figure 4, Table 3).

3.3. Comparison of Tenderness Threshold Value of the Tenderest Point in Diji (SP 8) Area. We used one-way ANOVA to analyze two groups' tenderness threshold value of the tenderest point in Diji (SP 8) area in menstrual and nonmenstrual period. The results showed $F=4.983, P=0.003<$ 0.05 , indicating that there was difference in threshold value between two groups in menstrual or nonmenstrual period. S-L-D method was used for further analysis. Tenderness threshold value in the menstrual period of the observation group was significantly lower than that in the nonmenstrual period, $P<P^{\prime}$, while there was no significant difference between two periods in the control group. In menstrual period, tenderness threshold value of the observation group was significantly lower than that of the control group, $P<P^{\prime}$. In nonmenstrual period, tenderness threshold value of the observation group was lower than that of the control group, while there was no significant difference, $P>P^{\prime}$ (Figure 5, Table 4).

3.4. Location of the Tenderest Point in Diji (SP 8) Area. Our results showed overlaps between the tenderest point and 
TABLE 2: Comparison of the TOR in Diji (SP 8) area between menstrual and nonmenstrual period.

\begin{tabular}{|c|c|c|c|c|c|c|}
\hline Group & & & Menstrual period & Nonmenstrual period & $x^{2}$ & $P$ \\
\hline \multirow{3}{*}{ Observation group } & \multirow{2}{*}{ Tenderness } & Positive & 53 & 38 & & \\
\hline & & Negative & 7 & 22 & 10.231 & 0.001 \\
\hline & \multicolumn{2}{|c|}{ TOR } & $88.3 \%$ & $63.3 \%^{*}$ & & \\
\hline \multirow{3}{*}{ Control group } & \multirow{2}{*}{ Tenderness } & Positive & 31 & 19 & & \\
\hline & & Negative & 29 & 41 & 4.937 & 0.026 \\
\hline & \multicolumn{2}{|c|}{ TOR } & $51.7 \%^{*}$ & $31.7 \%{ }^{\#}$ & & \\
\hline \multicolumn{3}{|l|}{$x^{2}$} & 19.206 & 12.063 & & \\
\hline \multicolumn{3}{|l|}{$P$} & 0.000 & 0.001 & & \\
\hline
\end{tabular}

Note: corrected value $P^{\prime}=0.00833 .{ }^{*}$ Compared with observation group in menstrual period, $P<P^{\prime} .{ }^{\#}$ Compared with observation group in nonmenstrual period, $P<P^{\prime}$.

TABLE 3: Comparison of VAS score between menstrual and nonmenstrual period in two groups ( $\mathrm{mm})$.

\begin{tabular}{|c|c|c|c|c|c|}
\hline Group & & Menstrual period & Nonmenstrual period & $Z$ & $P$ \\
\hline \multirow{5}{*}{ Observation group } & Number of effective values & 53 & 38 & \multirow{5}{*}{-2.646} & \multirow{5}{*}{0.0081} \\
\hline & Minimum value & 10 & 5 & & \\
\hline & Maximum value & 60 & 40 & & \\
\hline & $\mathrm{M}(\mathrm{QR})$ & $30(20)$ & $22.5(25)^{*}$ & & \\
\hline & Mean rank & 88.76 & 66.12 & & \\
\hline \multirow{5}{*}{ Control group } & Number of effective values & 31 & 19 & \multirow{7}{*}{-0.182} & \multirow{7}{*}{0.856} \\
\hline & Minimum value & 5 & 5 & & \\
\hline & Maximum value & 40 & 40 & & \\
\hline & $\mathrm{M}(\mathrm{QR})$ & $20(15)^{*}$ & $20(20)$ & & \\
\hline & Mean rank & 54.89 & 57.50 & & \\
\hline$Z$ & & -3.705 & -0.780 & & \\
\hline$P$ & & 0.000 & 0.436 & & \\
\hline
\end{tabular}

Note: corrected value $P^{\prime}=0.00833 .{ }^{*}$ Compared with observation group in menstrual period, $P<P^{\prime}$.

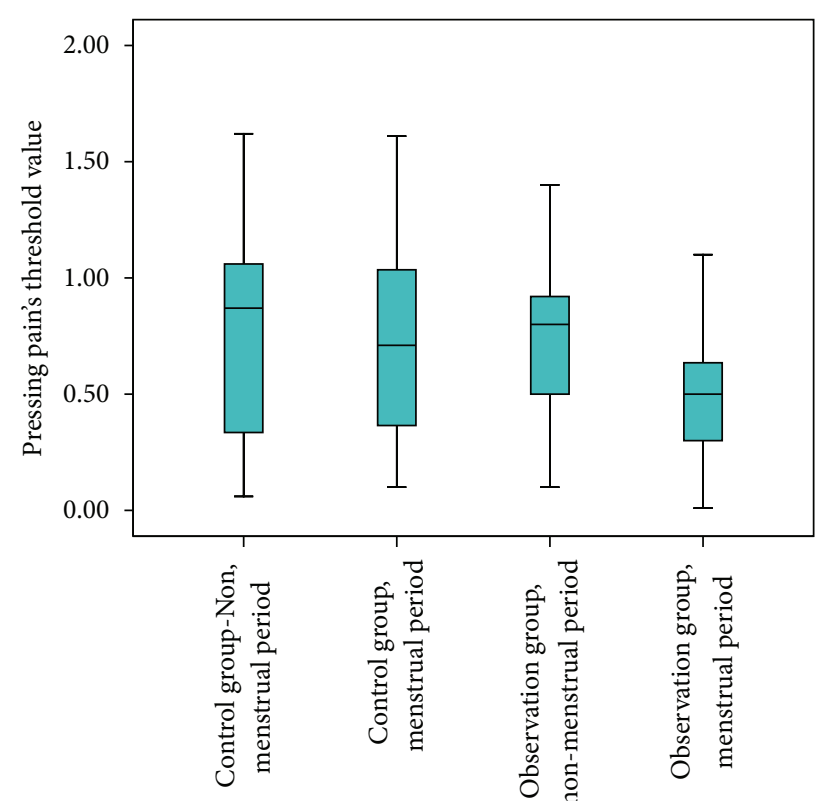

FIGURE 5: Tenderness threshold value in Diji (SP 8) area in menstrual and nonmenstrual period $(\mathrm{kPa})$. standard point in observation group during menstrual period and nonmenstrual period. Overlap rate was $22.6 \%$ and $28.9 \%$, respectively. We measured the distance between the tenderest point and the standard point and came up with the following conclusion: if we set standard point of Diji (SP 8) as datum point, distribution range of the tenderest point in observation group was $0.565-0.903 \mathrm{~cm}$ in menstrual period and $0.515-$ $0.974 \mathrm{~cm}$ in nonmenstrual period, respectively (Figure 6, Table 5).

\section{Discussions}

The dynamic characteristic of the acupuncture point is one of the hot topics in the acupuncture research. Previous study has shown that acupuncture points are of dimensional structure located in the interstice within the skin, vessel, muscle, sinew, bone, and even viscera, rather than fixed points. Their location may be influenced by several factors such as different physiological changes and pathological conditions of the Zang-fu organs and channels and the external environment and individual variety. Therefore, acupuncture points possess the individualized and dynamic characteristic [6]. 
TABLE 4: Tenderness threshold value in Diji (SP 8) area in menstrual and nonmenstrual period (kPa).

\begin{tabular}{lcccc}
\hline Group & & Menstrual period & Nonmenstrual period & Mean difference \\
\hline & Number of effective values & 53 & 38 & \\
Observation group & Minimum value & 0.01 & 0.1 & -0.251 \\
& Maximum value & 1.2 & 1.6 & 0.001 \\
& $\bar{x} \pm s$ & $0.497 \pm 0.040$ & 31 & $-0.375^{*}$ \\
Control group & Number of effective values & 31 & 0.06 & -0.027 \\
& Minimum value & 0.1 & 1.62 & 0.801 \\
Mean difference & Maximum value & 1.61 & $0.751 \pm 0.468$ & -0.003 \\
$P$ & $\bar{x} \pm s$ & $0.724 \pm 0.385^{*}$ & 0.979 & \\
\hline
\end{tabular}

Note: corrected value $P^{\prime}=0.00833$. ${ }^{*}$ Compared with observation group in menstrual period, $P<P^{\prime}$.

TABLE 5: Distance between the tenderest point and standard point in Diji (SP 8) area (cm).

\begin{tabular}{cccccccc}
\hline & & $\begin{array}{c}\text { Number of } \\
\text { effective values }\end{array}$ & $\begin{array}{c}\text { Overlap rate with } \\
\text { standard point }\end{array}$ & Minimum value & Maximum value & M (QR) & 95\% CI \\
\hline Observation group & Menstrual period & 53 & $22.6 \%$ & 0 & 3 & 0.70 .6 & $0.565-0.903$ \\
& Nonmenstrual period & 38 & $28.9 \%$ & 0 & 2.6 & 0.7 (1) & $0.515-0.974$ \\
\hline
\end{tabular}

There are four reasons for applying the pressing examination on Diji (SP 8) of the patients who suffered from primary dysmenorrheal to study the dynamism of acupuncture points on reflecting disease in this research. First, according to the channel and acupuncture point theory in TCM, Diji (SP 8) is the $X i$-cleft point of the Spleen meridian of Foot Taiyin. Xi-cleft points are where the meridian $q i$ accumulates deeply and are indicated for the acute and pain disease of the respective Zang-fu organs and meridians, while the $X i$ cleft points of the Yin meridians are also indicated for blood diseases [7]. Primary dysmenorrhea, a medical condition of cramping pain in the lower abdomen occurring just before and during menstruation, is ascribed to acute pain and blood disease in TCM. Therefore, Diji (SP 8) is the most important and commonly used point for the treatment of dysmenorrhea [8-11]. Second, the acupuncture points relate closely to the internal Zang-fu organs through the pathway of the meridians; thereby the condition of the diseased Zang$f u$ organs will be reflected on the acupuncture point through the transmission of the meridians [12]. Third, though there are various examination methods and techniques for the reflection effect of acupuncture point, such as detecting the electric currency and electrical resistance of the point [1317] and the infrared thermal imaging technique [18, 19], the most commonly used, convenient, and consensus method is detecting the tenderness and pain threshold [20-22]. Fourth, there are few researches and reports on reflecting effect of Diji (SP 8) on the dysmenorrhea.

Diji (SP 8) of the 30 patients with primary dysmenorrhea and 30 healthy women was palpated by hand and detected by algometer. The result of the research and the concerning issues are discussed as follows.

(1) The tenderness occurrence rates and the VAS score of Diji (SP 8) in menstrual period of the observation group were higher than that in nonmenstrual period and the menstrual period in the control group. The tenderness threshold of Diji (SP 8) in the menstrual period of observation group was lower than that in the nonmenstrual period of observation group and the menstrual period in the control group. The result showed that there exist dynamic characteristics in Diji (SP 8) in the tender reaction in both the physiological and pathological conditions, including the different stages in the pathological condition. The tenderness reaction was more likely to occur and more intensive and sensitive in the menstrual period of the primary dysmenorrhea patient.

(2) There is no statistical difference in the tenderness occurrence rates, VAS score, and tenderness threshold in both the menstrual period and nonmenstrual period in the control group. It indicates that there is no remarkable change in the tenderness reaction on Diji (SP 8) in the physiological state and the alteration of the physiological rhythm. Comparing the data in the nonmenstrual period of the test and control group, the tenderness occurrence rate of observation group is higher than that of the control group, but there is no statistical difference between the two groups in VAS value and the tenderness threshold. The results might be considered as follows: in the nonmenstrual period, the dysmenorrhea patients were still in the pathological states of blood deficiency failing to nourish the uterus or blood stasis blocking the meridian in the uterine, which makes Diji (SP 8) become more sensitive to pressing; however the severity was not intensive. Or it might be that the patients enrolled were not enough to show the statistical difference. From an anatomical 


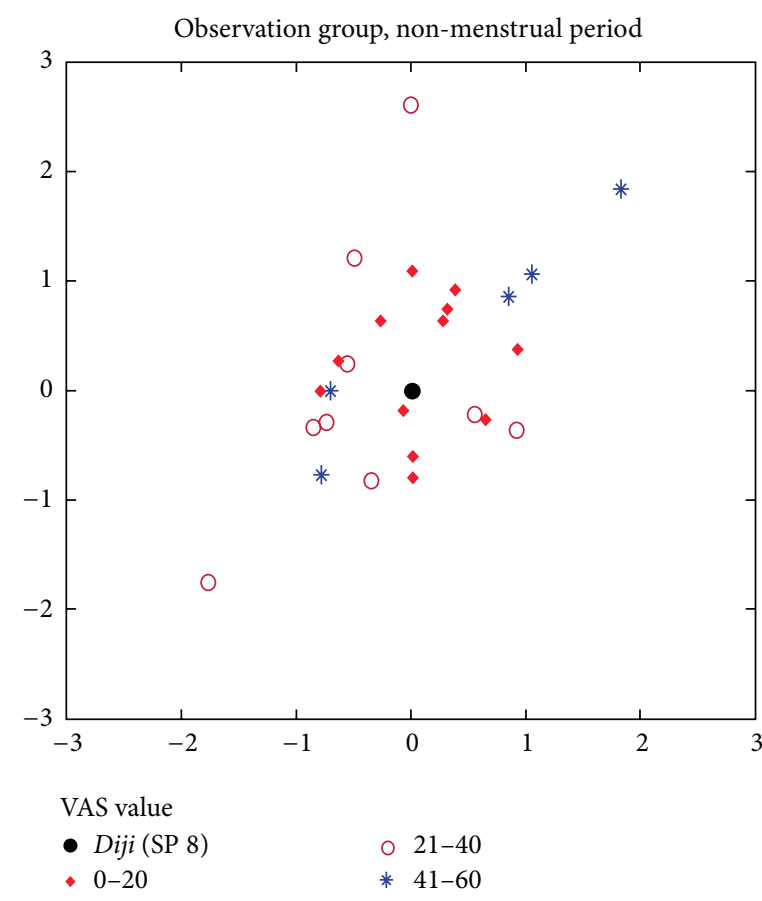

(a)

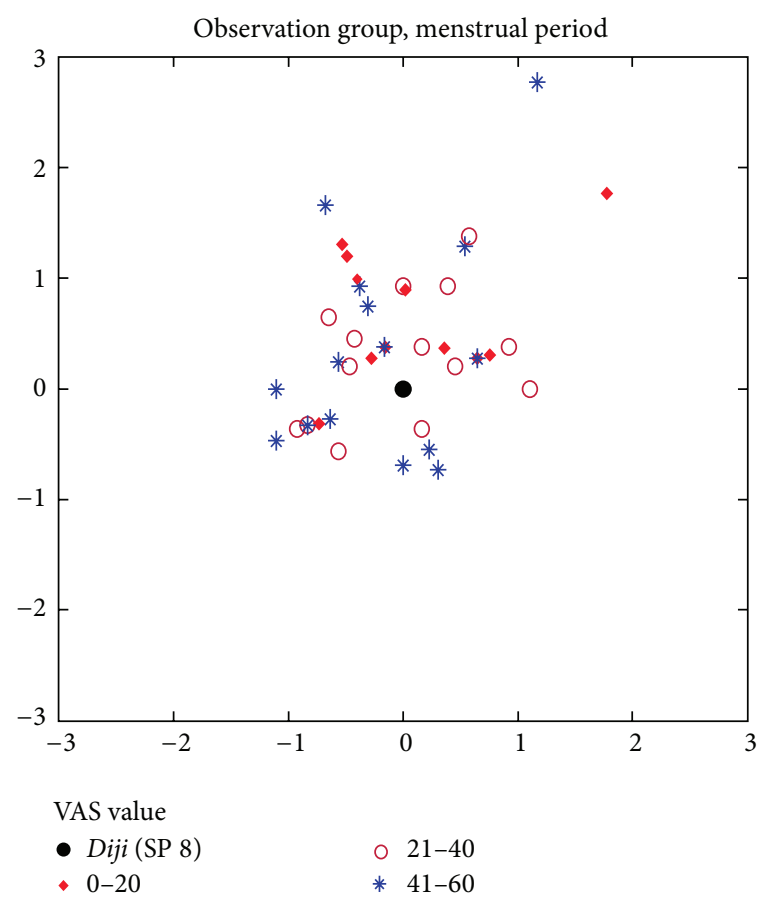

(b)

FIgURE 6: Location of the tenderest point in Diji (SP 8) area (cm).

perspective, there are parts of the saphenous nerve in shallow layer of Diji (SP 8) area and sympathetic nerve governing the myometrium contraction coming from the same nerve segments. Accordingly, the same situation also occurs between the tibial nerve in deep layer and parasympathetic nerve controlling the sense of uterus. It can thus be seen that Diji (SP 8) has a close relationship with uterus in anatomy [23]. However, the results still possibly indicate that Diji (SP 8) might reflect the pathological condition in the menstrual period of the dysmenorrhea patients, which also validate the viewpoint that pathological reaction in meridian and acupuncture points relates to timing [24].

(3) This research found out that, in Diji (SP 8) area, the tenderest points were divergent from the standardized location of Diji (SP 8) in the majority of dysmenorrheal patients, which indicates that the location of Diji (SP 8) in pathological state is different from the standardized location. Acupuncture point is both the reflective point of diseases and where the needles and moxibustion are applied for the treatment of disease in the meridian and acupuncture point theory [25]. Wang illuminates the process acupuncture reflecting disease. Acupuncture point is where both the pathological factors and meridian $q i$ exist when patient suffered from a disease. If the meridian $q i$ fails to dispel the pathological qi out of the body in time, the pathological factors accumulate in the acupuncture point; consequently, there might be pathological reactions such as pain, tenderness, and other changes on the acupuncture point [26]. Zeng et al. put forward the viewpoint that, in the pathological state, the surface reflection area of acupuncture varies with the condition of the disease; it increases when disease gets worse, decreases when disease gets better, and disappears when disease is cured [27, 28]. As to the enlargement of the surface areas around acupoint reflecting diseases, Yu et al. believe that it relates to the facilitation and sensitization of the spinal cord caused by visceral disorders, where the information coming from the body surface and viscera are assembled [29]. According to the theory of "painful locality taken as an acupoint," the tenderest point in Diji (SP 8) area might be the veracious point of Diji (SP 8) in the pathological state and it might also be the most effective point for treatment. We have found in clinic that, in the acute stage of dysmenorrhea, the part between Sanyinjiao (SP 6) and Yinlingquan (SP 9) on the pathway of Spleen meridian is the main reaction region. Tender, sore, or distending points, especially distinctive around the Sanyinjiao (SP 6), Diji (SP 8), and Yinlingquan (SP 9) area, will be found when palpating along the meridian. Needling on the tender point will bring instinctive effect of relieving the pain. Of course, large scale clinical trial is required to confirm our clinical observation.

(4) There have been 2 Chinese national standards of acupuncture location (1990 and 2006) and Standard Acupuncture Nomenclature (second edition) was 
published in 1993 [30-34]. The standardization of acupuncture point location helps to standardize the needle manipulation and also promote the spreading of acupuncture worldwide. In standardized acupuncture point location, a vertical and horizontal coordinate method is adopted as much as possible to locate the acupuncture point; for example, Zusanli (ST 36) is "on the anterior aspect of the leg, on the line connecting ST35 with ST41, 3 B-cun (proportional bone cun) inferior to ST35." Then, Zusanli (ST 36) is stated as the crossing point of the two intersecting lines. Accordingly, this locating method makes some acupuncture practitioners stick to the standardized location of acupoints and ignore the dynamic character of the acupoint. However, this might result in the less satisfied effect in clinic. The description of the location of some extra acupuncture point in textbook of acupuncture and moxibustion before the standardization of acupuncture point location in China embodies the dynamic character of acupuncture point. For example, Lanwei (appendix) point is located around 2 cun inferior to Zusanli (ST 36 ), and Danang (gallbladder) is 1-2 cun inferior to Yanglingquan (GB 34) [35].

(5) In acupuncture clinical trials, selecting the nontraditional-acupoint site near the acupoint for treatment as the placebo control is called adjacent nonacupoint control method [36], which is the main method in the nonacupoint controlled trials [37]. The way of selecting the nonacupoint is reasonable or does not determine the validity of the control and the authenticity and veracity of the results. This research as well as others [38-41] all revealed that the location and size of the acupoints may change with the different conditions of the body, and in the pathological condition the size on the body surface may even get large, which embodies the dynamic characteristic of the acupuncture points. Therefore, the adjacent nonacupoint might just be the site of acupuncture point for treatment, and needling the adjacent nonacupoint, possibly, might produce the same or even better effect compared to that of the acupuncture point in the standardized location. Thereby, the result of clinical trials adopting the nearby nonacupoint as a control is debatable.

This research is an exploratory study on the dynamism of acupuncture points through detecting the tenderness reaction on Diji (SP 8) in primary dysmenorrhea patients. Further studies are required for answering questions concerning the dynamism of acupuncture points, such as what the changes of the acupuncture points in different physiological and pathological conditions as well as in different stages of the pathological conditions are and whether or not needling on the tender site of the acupuncture point might improve the treatment effect.

\section{Conflict of Interests}

The authors declare that there is no conflict of interests regarding the publication of this paper.

\section{References}

[1] X. Y. Shen, Meridian and Acupoint, Traditional Chinese Medicine Publishing House, Beijing, China, 2007.

[2] G. Lefebvre, O. Pinsonneault, V. Antao et al., "Primary dysmenorrhea consensus guideline," Journal of Obstetrics and Gynaecology Canada, vol. 27, no. 12, pp. 1117-1146, 2005.

[3] D. J. Cox and R. G. Meyer, "Behavioral treatment parameters with primary dysmenorrhea," Journal of Behavioral Medicine, vol. 1, no. 3, pp. 297-310, 1978.

[4] Regional Office for the Western Pacific. WHO, WHO Standard Acupuncture Point Locations in the Western Pacific Region, World Health Organization, Western Pacific Region, 2008.

[5] M. K. Merboth and S. Barnason, "Managing pain: the fifth vital sign," The Nursing Clinics of North America, vol. 35, no. 2, pp. 375-383, 2000.

[6] M. F. Kang and R. X. Chen, "Theory of reaction point and acupoint," Journal of Jiangxi College of Traditional Chinese Medicine, vol. 18, no. 3, pp. 37-38, 2006.

[7] W. J. Li, "The case report of massage treatment on dysemenorrhea with needle syncope," China Foreign Medical Treatment, vol. 27, no. 33, p. 97, 2008.

[8] W. J. Yang, "Clinical observation of the theapeutic effect on 55 cases dysmenorrhea with string moxibustion of Zhuang Folk Medicine," Chinese Journal of Ethnomedicine and Ethnopharmacy, vol. 23, no. 1, p. 1, 2014.

[9] W. J. Li and C. Zhao, "Observation of the theapeutic effect on primary dysmenorrhea treated with needling cleft point-Diji," Shanghai Journal of Acupuncture and Moxibustion, vol. 30, no. 12, pp. 817-818, 2012.

[10] S. J. Li, Z. F. Li, S. Z. Gao et al., "Observation of the Theapeutic effect on moderate and severe primary dysmenorrhea patients' immediate pain ease effect by acupuncture," Jilin Journal of Traditional Chinese Medicine, vol. 32, no. 10, pp. 1060-1061, 2012.

[11] W. J. Li, Z. Y. Li, and B. Xiao, "Research on the transient analgesic effect of primary dysmenorrhea by press and rub Diji," Journal of Emergency in Traditional Chinese Medicine, vol. 21, no. 9, pp. 1392-1405, 2012.

[12] Z. S. Tan, The Practical Diagnosis with Meridians and Mcupoints, Science and Technology Publishing House, Guangdong, China, 2004.

[13] X. M. Fan, "Experimental research on measurement and application of meridians electrical impedance," Physics and Engineering, vol. 18, no. 3, pp. 41-42, 2012.

[14] W. S. Yang, "Study on low resistance of channels and collaterals," Journal of Beijing University (Natural Science), vol. 44, no. 2, pp. 281-288, 2008.

[15] X.-M. Wang, M. Luo, X.-L. Ma, P. Zhou, Z.-H. Wang, and S.Y. Ma, "Visibility of the meridian based on perfusion current," Chinese Journal of Biomedical Engineering, vol. 30, no. 3, pp. 472-480, 2011.

[16] Z. R. Li, Experimental Acupunture Science, China Press of Traditional Chinese Medicine, Beijing, China, 2004.

[17] A. P. Colbert, R. Hammerschlag, M. Aickin, and J. McNames, "Reliability of the prognos electrodermal device for measurements of electrical skin resistance at acupuncture points," 
Journal of Alternative and Complementary Medicine, vol. 10, no. 4, pp. 610-616, 2004.

[18] N. Tian, R. X. Chen, B. Xie et al., "Study of thermal sensitive acupoints' infrared radiation feature on bronchus asthma patients," Shanghai Journal of Acupuncture and Moxibustion, vol. 33, no. 2, pp. 174-176, 2012.

[19] J. Song, J. L. Wang, L. Xu et al., "Clinical efficacy on knee osteoarthritis based on evaluation of infrared thermal imaging technology on ashi points of meridian muscle region with acupuncture," World Journal of Integrated Traditional and Western Medicine, vol. 9, no. 9, pp. 948-950, 2014.

[20] C. H. Wang, K. H. Deng, and H. Zhu, "665 Investigations of acupoint's press-feel reaction on 58 holes," Chinese Journal of Basic Medicine in Traditional Chinese Medicine, vol. 20, no. 6, pp. 817-818, 2014.

[21] S. H. Wang, Q. Long, and Q. G. Liu, "The comparative observation of acupoint's pressure pain threshold on patients with gallbladder cardiac syndrome andcoronary heart disease," World Chinese Medicine, no. 8, pp. 1076-1078, 2014.

[22] H. Ben, L. Li, P.-J. Rong et al., "Observation of pain-sensitive points along the meridians in patients with gastric ulcer or gastritis," Evidence-Based Complementary and Alternative Medicine, vol. 2012, Article ID 130802, 7 pages, 2012.

[23] X. Zhang, Clinical Research of the Specificity of Diji-Point to Treat Primary Dysmenorrhea, Shandong University of Traditional Chinese Medicine, 2004.

[24] Q. F. Wu, J. M. Wu, X. H. Bai et al., "Back segment of du meridian tenderness reaction rule on patients with gastro-esophageal reflux disease," Chinese Acupuncture \& Moxibustion, vol. 34, no. 8, pp. 775-777, 2014.

[25] F. R. Liang and J. P. Zhao, Acupunture and Moxibustion Science, People's Medical Publishing House, Beijing, China, 2012.

[26] W. D. Wang, Zhen Dao Mo Xiang, China Press of Traditional Chinese Medicine, Beijing, China, 2011.

[27] F. Zeng, Y. Tang, X. G. Liu et al., "Discussion about the dynamics of acupoints," Journal of Sichuan of Traditional Chinese Medicine, vol. 26, no. 6, pp. 103-105, 2008.

[28] G. C. Gai, Chinese Acupoints Diagnostics, The Academy Press, Beijing, China, 1997.

[29] X. C. Yu, B. Zhu, J. H. Gao et al., "The science foundation of acupoints'dynamic process," Journal of Traditional Chinese Medicine, vol. 48, no. 11, pp. 971-973, 2007.

[30] D. S. Wang, Standard Acupuncture Nomenclature, World Health Organization, Regional Office for the Western Pacific, 1984.

[31] The State Bureau of Technology Supervision, National Standards of the People's Republic of China: Location of Acupoints, 1990.

[32] O. Akerele, "WHO and the development of acupuncture nomenclature: overcoming a tower of Babel," The American Journal of Chinese Medicine, vol. 19, no. 1, pp. 89-94, 1991.

[33] WHO, Standard Acupuncture Nomenclature: A Brief Explanation of 361 Classical Acupuncture Point Names and Their Multilingual Comparative List, World Health Organization, 1993.

[34] L. X. Huang, J. S. Zhao, and Z. Han, The Name and Location of Acupoints, 2006.

[35] M. L. Qiu, Acupunture and Moxibustion Science, Shanghai Science and Technology Press, Shanghai, China, 1985.

[36] Z.-S. Liu and Y.-Y. Cai, "Thinking on methodologies and problems existed in clinical study of acupuncture and moxibustion," Chinese Acupuncture \& Moxibustion, vol. 30, no. 1, pp. 67-71, 2010.
[37] X. G. Yang, Y. Y. Li, X. P. Tian et al., "The domestic and foreign research method review of acupuncture by the non acupoint and non meridian selection," Journal of Traditional Chinese Medicine, vol. 50, no. 8, pp. 748-750, 2009.

[38] Y. H. Miao, J. Yun, H. Y. Li et al., "Discussion about acuputure the nearby non acupoints with control method from the perspective of acupoints' dynamic characteristics," Journal of Traditional Chinese Medicine, vol. 55, no. 7, pp. 563-566, 2014.

[39] X. Z. Qi, C. F. Ji, H. Shi et al., "Distribution of sensitized acupoints on patients with functional intestinal disorder," World Chinese Medicine, no. 3, pp. 259-262, 2013.

[40] P.-J. Rong, S. Li, H. Ben et al., "Peripheral and spinal mechanisms of acupoint sensitization phenomenon," Evidence-Based Complementary and Alternative Medicine, vol. 2013, Article ID 742195, 6 pages, 2013.

[41] H. Shi, B. Cheng, J.-H. Li et al., "Mast cell and substance P are involved in the process of acupoint sensitization induced by acute gastric mucosal injury," Acupuncture Research, vol. 35, no. 5, pp. 323-329, 2010. 


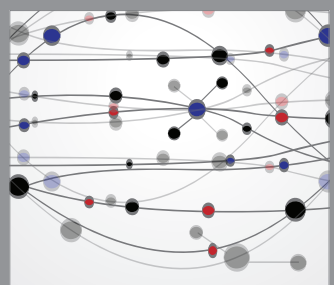

The Scientific World Journal
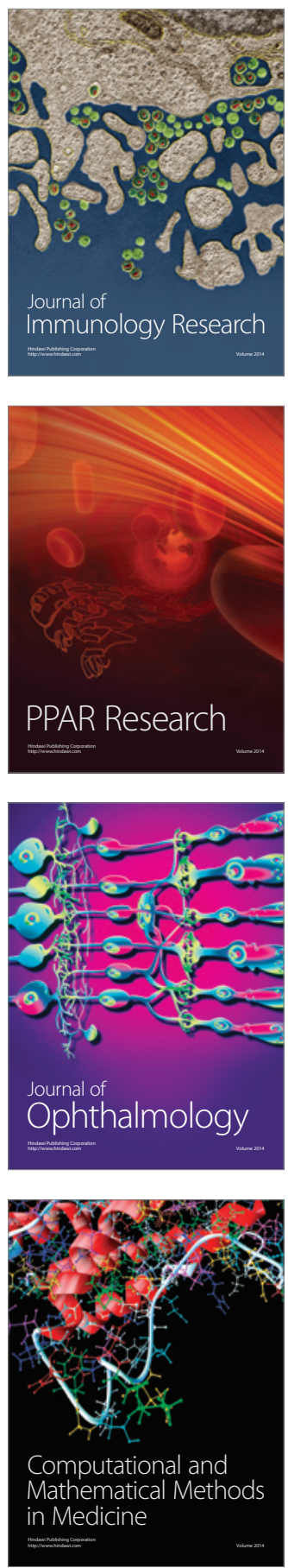

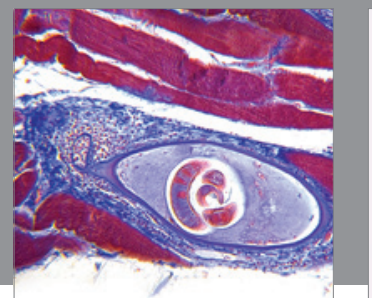

Gastroenterology

Research and Practice
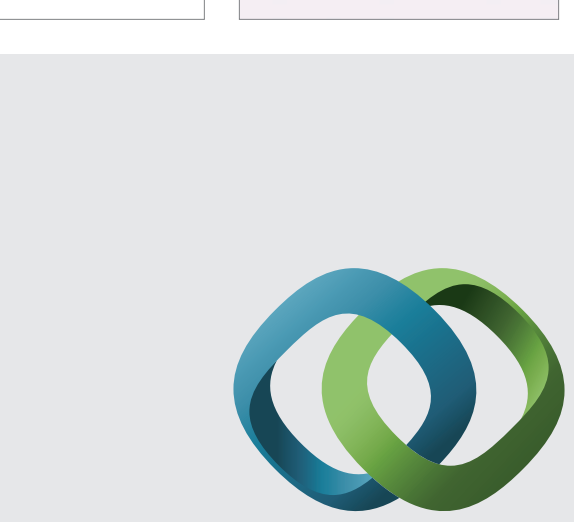

\section{Hindawi}

Submit your manuscripts at

http://www.hindawi.com
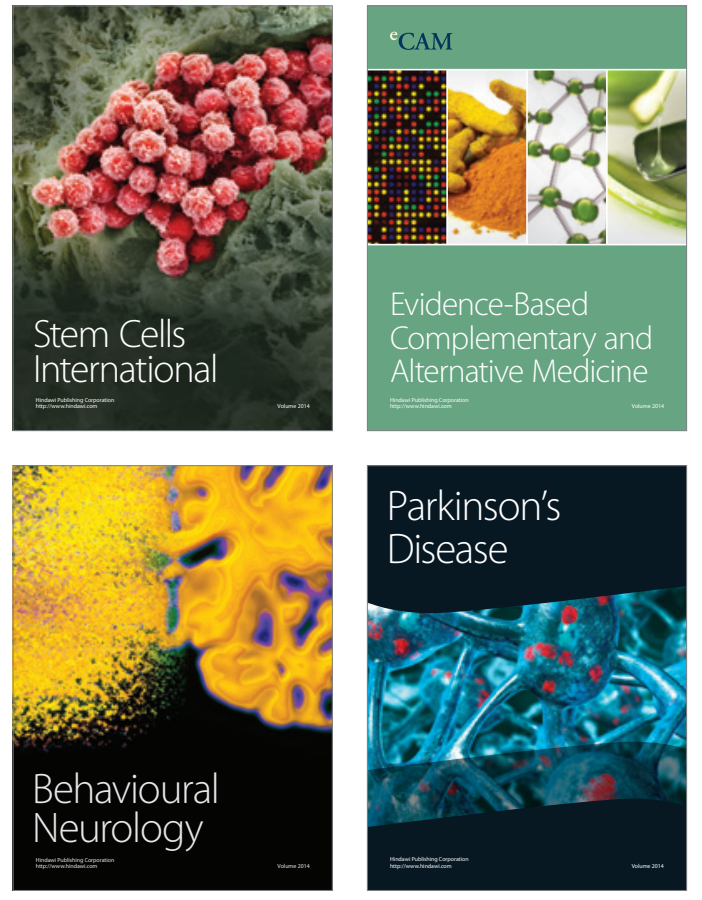
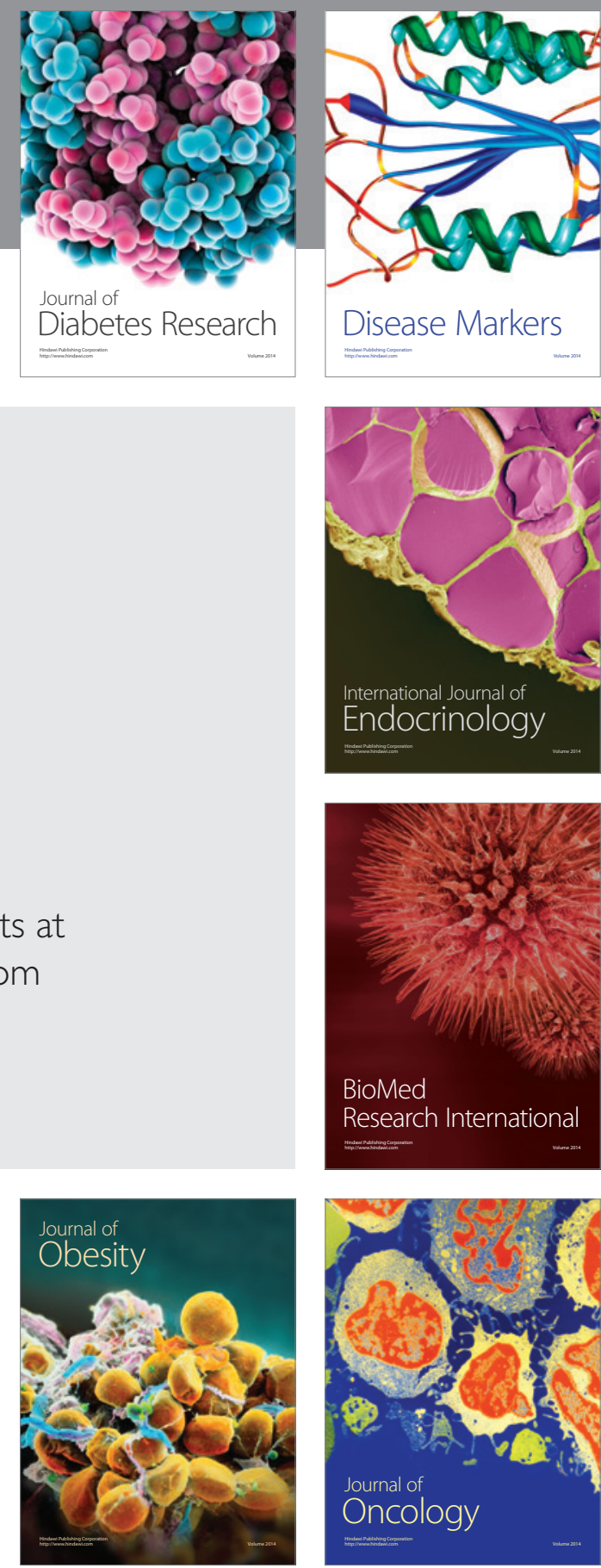

Disease Markers
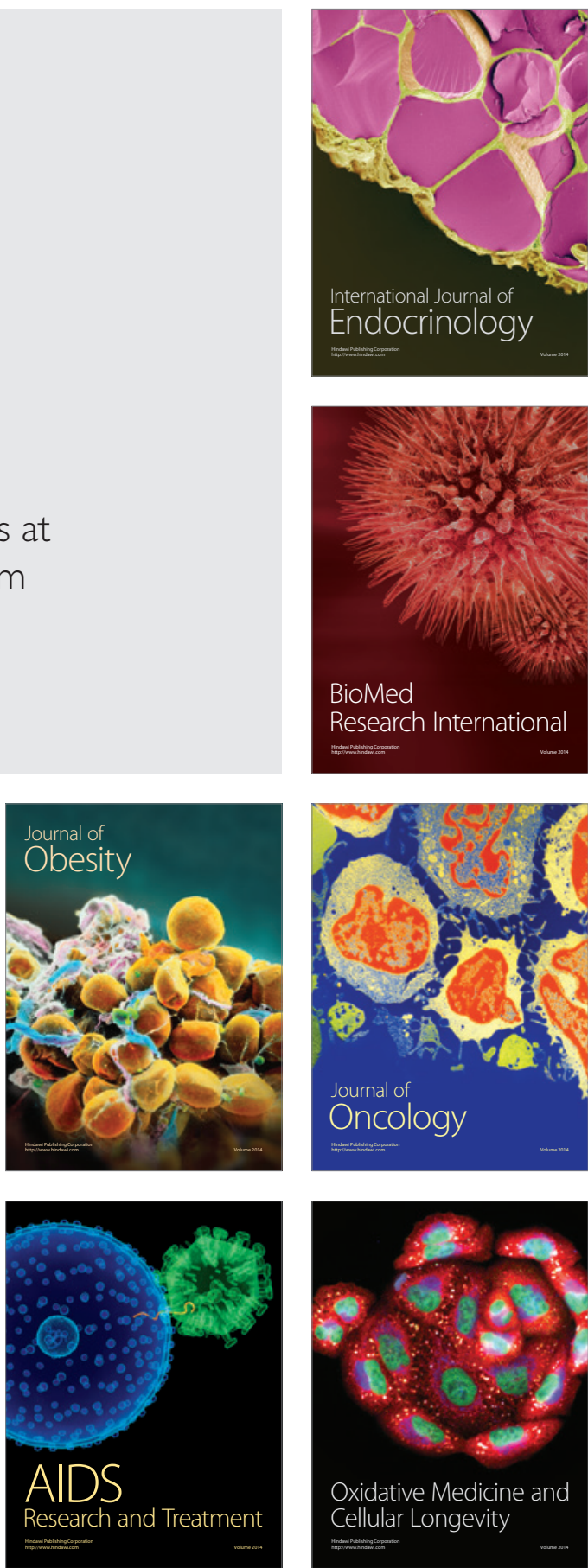